\title{
LENGTH MULTIPLICITIES OF HYPERBOLIC 3-MANIFOLDS
}

\author{
JOSEPH D. MASTERS
}

\begin{abstract}
Let $M=\mathbb{H}^{3} / \Gamma$ be a hyperbolic 3-manifold, where $\Gamma$ is a non-elementary Kleinian group. It is shown that the length spectrum of $M$ is of unbounded multiplicity.
\end{abstract}

\section{INTRODUCTION}

This paper is concerned with length multiplicities in hyperbolic 3-manifolds, or more generally, in hyperbolic 3-orbifolds. Let $M=$ $\mathbb{H}^{3} / \Gamma$ be a hyperbolic 3-orbifold, where $\Gamma$ is a non-elementary Kleinian group. We say that $\gamma \in \Gamma$ is loxodromic if $t r^{2} \gamma \notin[0,4]$ (note that this includes "hyperbolic" elements). Every loxodromic element $\gamma \in \Gamma$ has an associated complex length, denoted $\ell_{0}(\gamma)=\ell+i \theta$, which describes the action of $\gamma$ on $\mathbb{H}^{3}$ : along its invariant axis $\gamma$ translates a distance $\ell$ and rotates an angle $\theta$. We say that a complex length has multiplicity $n$ if it is shared by exactly $n$ conjugacy classes in $\Gamma$. We define the complex length spectrum, $L(M)$, to be the set of complex lengths of loxodromic elements of $\Gamma$, counted with multiplicity.

The real length spectrum of $M$ is defined to be the set of lengths of closed geodesics of $M$. The real length spectrum of $M$ is essentially the real part of $L(M)$ - the only difference being that the classes of $\gamma$ and $\gamma^{-1}$ are now equivalent.

Since $\Gamma \subset P S L_{2}(\mathbb{C}), \operatorname{tr}(\gamma)$ is well-defined, up to sign, for any $\gamma \in \Gamma$. The connection between traces and lengths is given by the following formula:

$$
\ell_{0}(\gamma)=2 \cosh ^{-1}\left(\frac{\operatorname{tr} \gamma}{2}\right) .
$$

Following [GR], we define, for any group $\Gamma$, the trace class of an element $\gamma \in \Gamma$ to be the set of elements $\gamma^{\prime} \in \Gamma$ for which $\operatorname{tr} \rho\left(\gamma^{\prime}\right)=\operatorname{tr} \rho(\gamma)$ for all representations $\rho: \Gamma \rightarrow S L_{2}(\mathbb{C})$. We define the stable multiplicity of $\gamma$ to be the number of conjugacy classes in the trace class of $\gamma$. Recall that a representation into $S L_{2}(\mathbb{C})$ is called irreducible if its image fixes no 1-dimensional subspaces of $\mathbb{C}^{2}$. 
We shall prove:

Theorem 1.1. Let $\Gamma$ be a finitely generated group which admits an infinite irreducible representation into $S L_{2}(\mathbb{C})$. Then $\Gamma$ has trace classes of unbounded stable multiplicity.

In Section 2 we prove that Theorem 1.1 has the following consequence:

Theorem 1.2. Let $M=\mathbb{H}^{3} / \Gamma$ be a hyperbolic 3-orbifold, where $\Gamma$ is a finitely generated, non-elementary Kleinian group. Then $L(M)$ is of unbounded multiplicity.

Corollary 1.3. If $M$ is a finite-volume, complete hyperbolic 3-manifold, then $L(M)$ is of unbounded multiplicity.

Corollary 1.4. If $M$ is a finite-volume, complete hyperbolic 3-manifold, then the real length spectrum of $M$ is of unbounded multiplicity.

The analogous statement for hyperbolic surfaces was proved by Randol (see $[R]$ ). We have reproduced the short proof in Section 3.

It is well-known that the length spectrum of an arithmetic hyperbolic 3-manifold has unbounded multiplicity. In fact, if $M$ is arithmetic, then the mean multiplicity, $n\left(\ell_{0}\right)$, of a length grows exponentially with $\ell_{0}$ (see $[\mathrm{M}]$ ).

Recent interest in length multiplicities of hyperbolic 3-manifolds has been sparked by connections with chaotic quantum systems. See [Sar] for more information.

I would like to thank Professors Fernando Rodriguez Villegas, John Tate and Cameron Gordon for useful conversations, Professor Peter Sarnak for valuable correspondence, the referee for helpful comments and Professor Alan Reid for his help and encouragement.

\section{Theorem 1.1 implies TheOREM 1.2}

In this section, we prove:

Claim: Theorem 1.1 implies Theorem 1.2

Proof of claim: By Selberg's Lemma, $\Gamma$ has a torsion-free subgroup $\Gamma^{\prime}$ of finite index $n$, say. Any set of more than $n$ elements which are pairwise non-conjugate in $\Gamma^{\prime}$ must contain at least two elements which are non-conjugate in $\Gamma$. Also, any representation of $\Gamma$ restricts to a representation of $\Gamma^{\prime}$. Therefore the stable multiplicities in $\Gamma^{\prime}$ will 
increase by at most a factor of $n$. So it is enough to consider the case where $\Gamma$ is torsion-free.

Let $\pi: S L_{2}(\mathbb{C}) \rightarrow P S L_{2}(\mathbb{C})$ be the natural projection. By $[\mathrm{T}]$, a non-elementary, torsion-free Kleinian group $\Gamma$ admits a faithful representation $\rho: \Gamma \rightarrow \pi^{-1}(\Gamma)$ such that $\pi \rho=i d$. Note that $\rho$ preserves trace (up to sign), and that $\rho$ is irreducible, since $\Gamma$ is non-elementary. Then, in the case that $\Gamma$ contains no parabolic elements, Theorem 1.2 is now an easy consequence of Theorem 1.1 and Equation 1.

In general, however, we must make sure that $\Gamma$ has trace classes with an unbounded number of loxodromic conjugacy classes. The claim will be proved once we show that any trace class in $\Gamma$ can contain only a bounded number of conjugacy classes of parabolic elements. This is done in the following lemma:

Lemma 2.1. Let $\Gamma$ be a finitely generated Kleinian group. Then there is an integer $N>0$ such that no trace class of $\Gamma$ contains more than $N$ conjugacy classes of parabolic elements. Moreover, if $\Gamma$ is geometrically finite, then a trace class of $\Gamma$ can contain at most two conjugacy class of parabolic elements.

The proof of this lemma will require some definitions.

Let $\Gamma$ be a finitely generated group. The space of characters, $V(\Gamma)$, is the set of all characters of representations of $\Gamma$ into $S L_{2}(\mathbb{C})$. By [CS], $V(\Gamma)$ has the structure of an affine algebraic set defined over $\mathbb{Q}$. The character of a representation $\rho$ is denoted $\chi_{\rho}$.

A quasi-conformal deformation of $\Gamma$ is a representation of $\Gamma$ into $P S L_{2}(\mathbb{C})$ which is induced by a quasiconformal homeomorphism of the Riemann sphere $\hat{\mathbb{C}}$. We say that a represntation of $\Gamma$ into $S L_{2}(\mathbb{C})$ is quasiconformal if it is the lift of a quasiconformal deformation of $\Gamma$ into $P S L_{2}(\mathbb{C})$.

Proof. (of Lemma 2.1) Using Selberg's Lemma as above, we may assume $\Gamma$ is torsion-free, so the identity representation lifts to a representation $\rho_{0}: \Gamma \rightarrow S L_{2}(\mathbb{C})$.

By the compact core theorem $([\mathrm{Sc}]), \Gamma$ can contain only finitely many conjugacy classes of maximal parabolic subgroups. Let

$\alpha_{1}, \beta_{1}, \ldots, \alpha_{n}, \beta_{n}$ generate the conjugacy classes of rank 2 maximal parabolic subgroups and $\gamma_{1}, \ldots, \gamma_{m}$ generate the conjugacy classes of rank 1 maximal parabolic subgroups.

First, suppose $\Gamma$ is finite covolume, so there are no $\gamma_{i}$ 's. We shall handle this case with Thurston's hyperbolic Dehn surgery theory. 
Let $V_{0}(\Gamma)$ denote the irreducible component of $V(\Gamma)$ containing $\chi_{\rho_{0}}$. Consider the map $\tau: V_{0}(\Gamma) \rightarrow \mathbb{C}^{n}$ defined by $\tau\left(\chi_{\rho}\right)=\left(\chi_{\rho}\left(\alpha_{1}\right), \chi_{\rho}\left(\alpha_{2}\right), \ldots, \chi_{\rho}\left(\alpha_{n}\right)\right)=\left(\operatorname{tr} \rho\left(\alpha_{1}\right), \operatorname{tr} \rho\left(\alpha_{2}\right), \ldots, \operatorname{tr} \rho\left(\alpha_{n}\right)\right)$. By Chapter 5 of [T], the image of $\tau$ covers an open neighborhood $U$ of $(2,2, \ldots, 2)$. So given two parabolic elements on distinct cusps, we can make one loxodromic while the other remains parabolic. In particular, for any $i \neq j$, and any integers $m_{1}, n_{1}, m_{2}, n_{2}$ (not all 0 ) there is a representation $\rho$ for which $\operatorname{tr} \rho\left(\alpha_{i}^{m_{1}} \beta_{i}^{n_{1}}\right) \neq \operatorname{tr} \rho\left(\alpha_{j}^{m_{2}} \beta_{j}^{n_{2}}\right)$, so $\alpha_{i}^{m_{1}} \beta_{i}^{n_{1}}$ and $\alpha_{j}^{m_{2}} \beta_{j}^{n_{2}}$ are not in the same trace class.

Now suppose we are given two distinct, non-trivial parabolic elements $\alpha_{i}^{m_{1}} \beta_{i}^{n_{1}}$ and $\alpha_{i}^{m_{2}} \beta_{i}^{n_{2}}$ on the same cusp. Suppose also that the elements are not in the same cyclic subgroup. Then Thurston's hyperbolic Dehn surgery theory shows that there is a representation taking one of the elements to the identity and the other to a loxodromic element, so they are in distinct trace classes. If the two elements are in the same cyclic subgroup, then by mapping them to loxodromics we see that they are in distinct trace classes, provided they are not inverses of each other.

It follows that we can find characters of representations in $\tau^{-1}(U)$ which differ on any distinct pair of parabolic elements in $\Gamma$ which are not inverses of each other, concluding the case where $\Gamma$ has finite covolume.

If $\Gamma$ is geometrically finite, then by $[\mathrm{Br}]$ there is a quasi-conformal deformation $\Gamma^{\prime}$ of $\Gamma$ and a finite covolume Kleinian group $\Gamma^{*}$ for which $\Gamma^{\prime} \subset \Gamma^{*}$. Since a quasi-conformal deformation takes parabolics to parabolics, the proof of this case now follows from the proof of the finite covolume case.

In general, if $\Gamma$ is any finitely generated Kleinian group, then $\Gamma$ has a faithful discrete representation $\rho$ for which $\rho(\Gamma)$ is geometrically finite, $\rho\left(\alpha_{i}\right)$ and $\rho\left(\beta_{i}\right)$ are parabolic for all $i$, and $\rho\left(\gamma_{i}\right)$ is loxodromic for all $i$; this is Thm 2.3 of $[\mathrm{A}]$ and follows from the Scott core theorem and the Thurston uniformization theorem. By the geometrically finite case, any two elements of the form $\alpha_{i}^{r} \beta_{j}^{s}$ in the same trace class of $\Gamma$ must be inverses of each other. So a trace class of $\Gamma$ can contain at most two elements of the form $\alpha_{i}^{r} \beta_{j}^{s}$. And since $\rho\left(\gamma_{i}\right)$ is loxodromic, $\operatorname{tr} \rho\left(\gamma_{i}^{r}\right) \neq \operatorname{tr} \rho\left(\gamma_{i}^{s}\right)$ if $|r| \neq|s|$, so $\gamma_{i}^{r}$ and $\gamma_{i}^{s}$ are not in the same trace class in $\Gamma$, and a trace class in $\Gamma$ can contain at most $2 m$ elements of the form $\gamma_{i}^{r}$ (as $i$ goes from 1 to $m$ ). Therefore a trace class in $\Gamma$ can contain at most $2(m+1)$ conjugacy classes of parabolic elements, and the lemma is proved. 


\section{BaCkground AND THE IDEA OF THE PROOF}

Suppose $\rho$ is an irreducible representation of $\Gamma$ into $S L_{2}(\mathbb{C})$. We first must find elements in $\rho(\Gamma)$ with the same trace. This can be done as in $[\mathrm{H}]$, with the aid of simple trace identities. For example, it is proved in $[\mathrm{H}]$ that $\operatorname{tr}\left(a^{2} b a b^{-1}\right)=\operatorname{tr}\left(b a^{2} b^{-1} a\right)$ for any elements $a$ and $b$ in $S L_{2}(K)$, where $K$ is any field. Then $a^{2} b a b^{-1}$ and $b a^{2} b^{-1} a$ are in the same trace class in $\Gamma$. In Section 4 , we will use these identities to construct sequences of words in $\Gamma$, all in the same trace class.

The problem, then, is to show that these words are not conjugate in $\Gamma$. This is done by finding a homomorphism of $\Gamma$ onto a finite group $G$, and showing that the images of the words are not conjugate in $G$.

This technique is nicely illustrated in the 2-dimensional case. The proof we give of the following theorem is a slight modification of the one which appears in $[\mathrm{R}]$.

Theorem 3.1. (Randol) Let $M=\mathbb{H}^{2} / \Gamma$ be a finite-volume hyperbolic surface. Then $\Gamma$ contains trace classes of unbounded stable multiplicity, and $L(M)$ contains lengths of unbounded multiplicity.

Proof. First, let us assume $M$ is compact.

$\Gamma \subset P S L_{2}(\mathbb{R})$ is a Fuchsian group, which can be embedded into $S L_{2}(\mathbb{R})$ so that traces are preserved up to sign. Then by Equation 1, it is enough to prove that $\Gamma \subset S L_{2}(\mathbb{R})$ has trace classes of unbounded stable multiplicity.

$\Gamma$ has the standard presentation:

$$
\Gamma=<a_{1}, b_{1}, a_{2}, b_{2}, \ldots, a_{g}, b_{g} \mid\left(a_{1} b_{1} a_{1}^{-1} b_{1}^{-1}\right) \ldots\left(a_{g} b_{g} a_{g}^{-1} b_{g}^{-1}\right)=1>.
$$

Note that there is a natural surjection

$$
\phi: \Gamma \rightarrow<a_{1}>*<a_{2}>* \ldots *<a_{g}>.
$$

In particular, $a_{1}$ and $a_{2}$ generate a free group $F$.

It follows from $[\mathrm{H}]$ that if $x$ and $y$ freely generate a free subgroup $F$ of $\Gamma$, then for any $\mathrm{N}$ there are words $w_{1}, \ldots, w_{N}$ in $x$ and $y$ such that

1. $w_{i}(x, y)$ and $w_{j}(x, y)$ are in the same trace class $\forall i, j \leq N$

2. $w_{i}(x, y)$ is not conjugate in $F$ to $w_{j}(x, y) \forall i \neq j$.

Consider the words $w_{1}\left(a_{1}, a_{2}\right), \ldots, w_{N}\left(a_{1}, a_{2}\right)$. By 1 , we have $w_{i}\left(a_{1}, a_{2}\right)$ and $w_{j}\left(a_{1}, a_{2}\right)$ are in the same trace class $\forall i, j$. By 2 , we have that any distinct pair $w_{i}\left(a_{1}, a_{2}\right), w_{j}\left(a_{1}, a_{2}\right)$ are not conjugate in $F$, hence their images are not conjugate in $<a_{1}>* \ldots *<a_{g}>$, 
hence $w_{i}\left(a_{1}, a_{2}\right)$ and $w_{j}\left(a_{1}, a_{2}\right)$ are not conjugate in $\Gamma$. This proves the theorem in the compact case.

If $M$ is non-compact, then $\Gamma$ is free. Then the same proof works to show that $\Gamma$ contains trace classes of unbounded stable multiplicity. The only complication is in passing to the statement about $L(M)$; for the elements of the trace class may be parabolic. However, $\Gamma$ admits faithful representations into $S L_{2}(\mathbb{C})$ for which every element becomes loxodromic, and therefore, as in the proof of Lemma 2.1, we see that a trace class can contain at most $2 n$ conjugacy classes of parabolic elements, where $n$ is the number of cusps, and the result follows.

The proof in three dimensions is more complicated, as hyperbolic 3-manifold groups do not generally surject onto non-abelian free groups. For example, if the manifold has zero first Betti number, such as a non-zero surgery on the figure-eight knot, then its fundamental group cannot surject onto any free group.

However, hyperbolic 3-manifold groups do surject onto groups of the form $P S L_{2}\left(\mathbb{F}_{p^{i}}\right)$, where $\mathbb{F}_{p^{i}}$ denotes the finite field of order $p^{i}$. In Sections 5 and 6 , we shall review the construction of these homomorphisms.

So the idea is to use $[\mathrm{H}]$ to construct a sequence of words $w_{i}(a, b)$ in the free group $F$ on $a$ and $b$ which are not conjugate in $F$ but which are in the same trace class of $F$. We map these words into $\Gamma$; their images will be in the same trace class of $\Gamma$. Then we map the words from $\Gamma$ into a group of the form $P S L_{2}\left(\mathbb{F}_{p}\right)$, and hope that these images will be non-conjugate in $P S L_{2}\left(\mathbb{F}_{p}\right)$, so the words will be non-conjugate in $\Gamma$. However, by $[H]$, the traces of the images are equal (up to sign) in $P S L_{2}\left(\mathbb{F}_{p}\right)$, and it is nearly true that two elements of $P S L_{2}\left(\mathbb{F}_{p}\right)$ are conjugate if and only if they have the same trace. Therefore care is needed in the choice of the words and the primes.

\section{TRACE IDENTITIES}

All of the trace identities which we shall use are ultimately based on the following lemma.

Lemma 4.1. (Horowitz) Suppose $K$ is a field, $a, b \in S L_{2}(K)$ with $\operatorname{tr} a=\operatorname{tr} b$, and $W(x, y)$ is a word in $x$ and $y$. Then $\operatorname{tr}(W(a, b))=\operatorname{tr}(W(b, a))$. 
Proof. It is proved in $[\mathrm{H}]$ (see also $[\mathrm{CS}]$ ) that there is a 3 -variable polynomial $P$ over $K$ such that $\operatorname{tr}(W(a, b))=P(\operatorname{tr} a, \operatorname{tr} b, \operatorname{tr} a b)$, for any elements $a, b \in S L_{2}(K)$. Then $\operatorname{tr}(W(b, a))=P(\operatorname{tr} b, \operatorname{tr} a, \operatorname{tr} b a)$. We have assumed that $\operatorname{tr} a=\operatorname{tr} b$, and for any matrices in $S L_{2}(K)$, $\operatorname{tr} a b=\operatorname{tr} b a$, so the lemma follows.

In Section 3, we gave the example $\operatorname{tr}\left(a^{2} b a b^{-1}\right)=\operatorname{tr}\left(b a^{2} b^{-1} a\right)$ for any elements $a, b \in S L_{2}(K)$. This follows by setting $W(x, y)=x^{2} y$ and noting that $\operatorname{tr}\left(W\left(a, b a b^{-1}\right)\right)=\operatorname{tr}\left(W\left(b a b^{-1}, a\right)\right)$ by Lemma 4.1.

We shall now construct the required elements of the same trace in $\rho(\Gamma)$ which we need to prove Theorem 1.1. We remark that one can construct much simpler sequences of elements of equal trace; however, in Section 6 we shall require the words to be of this special form in order to prove they are non-conjugate.

We now recursively define words $w_{n, i}$, for $i \leq n+1$. In what follows, we routinely supress the dependence of these words on $a, b, p_{i}$, $q_{i}$ and $k_{i}$; we will explain how to choose them later.

Let $W_{n}(x, y)=\left(x^{p_{n}-1+q_{n}} y^{-q_{n}}\right)^{k_{n}} x\left(x^{p_{n}-1+q_{n}} y^{-q_{n}}\right) x^{-1}$

$$
\begin{aligned}
\bar{W}_{n}(x, y)=x\left(x^{p_{n}-1+q_{n}} y^{-q_{n}}\right)^{k_{n}} x^{-1}\left(x^{p_{n}-1+q_{n}} y^{-q_{n}}\right) \\
\begin{aligned}
w_{1,1} & =W_{1}(a, b) \\
& =\left(a^{p_{1}-1+q_{1}} b^{-q_{1}}\right)^{k_{1}} a\left(a^{p_{1}-1+q_{1}} b^{-q_{1}}\right) a^{-1} \\
w_{1,2} & =\bar{W}_{1}(a, b) \\
& =a\left(a^{p_{1}-1+q_{1}} b^{-q_{1}}\right)^{k_{1}} a^{-1}\left(a^{p_{1}-1+q_{1}} b^{-q_{1}}\right) \\
w_{2,1} & =W_{2}\left(w_{1,1}, w_{1,2}\right) \\
& =\left(w_{1,1}^{p_{2}-1+q_{2}} w_{1,2}^{-q_{2}}\right)^{k_{2}} w_{1,1}\left(w_{1,1}^{p_{2}-1+q_{2}} w_{1,2}^{-q_{2}}\right) w_{1,1}^{-1} \\
w_{2,2} & =\bar{W}_{2}\left(w_{1,1}, w_{1,2}\right) \\
& =w_{1,1}\left(w_{1,1}^{p_{2}-1+q_{2}} w_{1,2}^{-q_{2}}\right)^{k_{2}} w_{1,1}^{-1}\left(w_{1,1}^{p_{2}-1+q_{2}} w_{1,2}^{-q_{2}}\right) \\
w_{2,3} & =W_{2}\left(w_{1,2}, w_{1,1}\right) \\
& =\left(w_{1,2}^{p_{2}-1+q_{2}} w_{1,1}^{-q_{2}}\right)^{k_{2}} w_{1,2}\left(w_{1,2}^{p_{2}-1+q_{2}} w_{1,1}^{-q_{2}}\right) w_{1,2}^{-1}
\end{aligned}
\end{aligned}
$$

Assume that $w_{n-1, i}$ has been defined for $i \leq n$, and that $w_{n-1,1}$ and $w_{n-1,2}$ are both words in $w_{n+1-i, 1}$ and $w_{n+1-i, 2}$ for each $i$ with 
$3 \leq i \leq n$ (note that this property is vacuous for $n=2$, the base case of the recursion).

Define $w_{n, 1}=W_{n}\left(w_{n-1,1}, w_{n-1,2}\right)$

$w_{n, 2}=\bar{W}_{n}\left(w_{n-1,1}, w_{n-1,2}\right)$

We claim that $w_{n, 1}$ and $w_{n, 2}$ are both words in $w_{n+2-i, 1}$ and $w_{n+2-i, 2}$ for $3 \leq i \leq n+1$.

Indeed, since $w_{n-1,1}$ and $w_{n-1,2}$ are words in $w_{n+1-i, 1}$ and $w_{n+1-i, 2}$ for $3 \leq i \leq n$, then $w_{n, 1}$ and $w_{n, 2}$ are both words in $w_{n+2-i, 1}$ and $w_{n+2-i, 2}$ for $4 \leq i \leq n+1$. And for $i=3$, it is obvious that $w_{n, 1}$ and $w_{n, 2}$ are words in $w_{n-1,1}$ and $w_{n-1,2}$.

Then we define:

$w_{n, i}=w_{n, 1}^{[i]}$ for $3 \leq i \leq n+1$,

where, if $w_{n, j}$ is a word in $w_{n+2-i, 1}$ and $w_{n+2-i, 2}, w_{n, j}^{[i]}$ denotes the word obtained by switching $w_{n+2-i, 1}$ and $w_{n+2-i, 2}$.

This gives a well-defined sequence of words $w_{n, i}$ for any positive integers $n, i \leq n+1$.

The following formulas, which are just formal consequences of our notation, will be useful:

$$
\begin{aligned}
w_{n, 3} & =w_{n, 1}^{[3]} \\
& =W_{n}\left(w_{n-1,2}, w_{n-1,1}\right) \\
& =\left(w_{n-1,2}^{p_{n}-1+q_{n}} w_{n-1,1}^{-q_{n}}\right)^{k_{n}} w_{n-1,2}\left(w_{n-1,2}^{p_{n}-1+q_{n}} w_{n-1,1}^{-q_{n}}\right) w_{n-1,2}^{-1} \text { for } n \geq 2 . \\
w_{n, i} & =w_{n, 1}^{[i]} \\
& =W_{n}\left(w_{n-1,1}^{[i-1]}, w_{n-1,2}^{[i-1]}\right) \\
& =\left[\left(w_{n-1,1}^{[i-1]}\right)^{p_{n}-1+q_{n}}\left(w_{n-1,2}^{[i-1]}\right)^{-q_{n}}\right]^{k_{n}} \\
& \quad w_{n-1,1}^{[i-1]}\left[\left(w_{n-1,1}^{[i-1]}\right)^{p_{n}-1+q_{n}}\left(w_{n-1,2}^{[i-1]}\right)^{-q_{n}}\right]\left(w_{n-1,1}^{[i-1]}\right)^{-1},
\end{aligned}
$$

for $n \geq 3$ and $4 \leq i \leq n+1$.

Regardless of the choices of $a, b, p_{i}$ and $q_{i}$, we have: 
Proposition 4.2. Let $K$ be a field, and let $a, b \in S L_{2}(K)$. Then $\operatorname{tr}\left(w_{i, j}\right)=\operatorname{tr}\left(w_{i, k}\right)$ for all $j, k \leq i+1$.

We shall require the following lemma:

Lemma 4.3. $\operatorname{tr}\left(W_{n}(x, y)\right)=\operatorname{tr}\left(\bar{W}_{n}(x, y)\right)$ for any $n$ and any $x, y \in K$.

Proof. Letting $U_{n}(x, y)=x^{k_{n}} y$, we have

$$
\begin{aligned}
\operatorname{tr}\left(W_{n}(x, y)\right) & =\operatorname{tr}\left(U_{n}\left(x^{p_{n}-1+q_{n}} y^{-q_{n}}, x\left(x^{p_{n}-1+q_{n}} y^{-q_{n}}\right) x^{-1}\right)\right) \\
& =\operatorname{tr}\left(U_{n}\left(x\left(x^{p_{n}-1+q_{n}} y^{-q_{n}}\right) x^{-1}, x^{p_{n}-1+q_{n}} y^{-q_{n}}\right)\right) \\
& =\operatorname{tr}\left(\bar{W}_{n}(x, y)\right) .
\end{aligned}
$$

Proof. (of Prop. 4.2)

We proceed by induction. By Lemma 4.3, $\operatorname{tr}\left(w_{1,1}\right)=\operatorname{tr}\left(w_{1,2}\right)$.

Now, suppose that $\operatorname{tr}\left(w_{n-1, i}\right)=\operatorname{tr}\left(w_{n-1, j}\right)$ for all $i, j \leq n$.

By Lemma 4.3, $\operatorname{tr}\left(w_{n, 1}\right)=\operatorname{tr}\left(w_{n, 2}\right)$. For $3 \leq i \leq n+1$, recall that $w_{n, 1}=U\left(w_{n+2-i, 1}, w_{n+2-i, 2}\right)$ for some word $U$; therefore we have:

$$
\begin{aligned}
\operatorname{tr}\left(w_{n, 1}\right) & =\operatorname{tr}\left(U\left(w_{n+2-i, 1}, w_{n+2-i, 2}\right)\right) \text { for some word } U \\
\operatorname{tr}\left(w_{n, i}\right) & =\operatorname{tr}\left(w_{n, 1}^{[i]}\right) \\
& =\operatorname{tr}\left(U\left(w_{n+2-i, 2}, w_{n+2-i, 1}\right)\right) .
\end{aligned}
$$

By the inductive hypothesis, $\operatorname{tr}\left(w_{n+2-i, 1}\right)=\operatorname{tr}\left(w_{n+2-i, 2}\right)$, so $\operatorname{tr}\left(w_{n, 1}\right)=\operatorname{tr}\left(w_{n, i}\right)$, by Lemma 4.1.

\section{Algebraic Representations}

The existence of maps from $\Gamma$ onto the groups $P S L_{2}\left(\mathbb{F}_{p_{i}}\right)$ depends on the existence of an algebraic representation of $\Gamma$, defined as follows:

$\rho: \Gamma \rightarrow S L_{2}(\mathbb{C})$ is an algebraic representation if it is irreducible and its image is an infinite subgroup of $S L_{2}(\overline{\mathbb{Q}})$, where $\overline{\mathbb{Q}}$ is the algebraic closure of $\mathbb{Q}$. Note that this differs slightly from the definition given in $[\mathrm{LR}]$.

The purpose of this section is to show that any group $\Gamma$ satisfying the hypotheses of Theorem 1.1 admits an algebraic representation.

Lemma 5.1. Let $\Gamma$ be a finitely generated group which admits an infinite irreducible representation $\rho_{0}: \Gamma \rightarrow S L_{2}(\mathbb{C})$. Then $\Gamma$ admits an algebraic representation. 
Proof. (of Lemma 5.1)

Let $V_{0}(\Gamma)$ be the irreducible component of $V(\Gamma)$ containing the character $\chi_{\rho_{0}}$ of the representation $\rho_{0}$ (recall the definition of $V(\Gamma)$ in Section 2).

If $\operatorname{dim}\left(V_{0}(\Gamma)\right)=0$, then it is a well known fact that the coordinates of $\chi_{\rho_{0}}$ must be algebraic; in other words the image of $\chi_{\rho_{0}}$ must lie in $\overline{\mathbb{Q}}$. Then it follows from $[\mathrm{Ba}]$ that $\rho_{0}$ is conjugate to an algebraic representation.

So suppose $\operatorname{dim}\left(V_{0}(\Gamma)\right)>0$. For $\gamma \in \Gamma$, define the function $\tau_{\gamma}$ : $V_{0}(\Gamma) \rightarrow \mathbb{C}$ by $\tau_{\gamma}\left(\chi_{\rho}\right)=\chi_{\rho}(\gamma)=\operatorname{tr} \rho(\gamma)$. Recall, by $[\mathrm{H}]$ or $[\mathrm{CS}]$, that a character is determined by the values it takes on a finite set of elements $\gamma_{1}, \ldots, \gamma_{n} \in \Gamma$. Then since $\operatorname{dim}\left(V_{0}(\Gamma)\right)>0$, there is some $\gamma_{i}$ for which $\tau_{\gamma_{i}}$ is non-constant; let us assume it is $\gamma_{1}$. Since $\tau_{\gamma_{1}}$ is a nonconstant polynomial map, it is surjective. Now, all the characters in some neighborhood $U$ of $\chi_{\rho_{0}}$ will correspond to infinite irreducible representations. $\tau_{\gamma_{1}}(U)$ is an open set in $\mathbb{C}$, and therefore contains an algebraic number $\alpha$. Let $\rho_{1} \in \tau_{\gamma_{1}}^{-1}(\alpha) ; \rho_{1}$ is infinite and irreducible, because it is in $U$.

Suppose $\alpha$ has a minimal polynomial $f$ with coefficients in $\mathbb{Z}$. Consider the algebraic subset $A_{1}(\Gamma) \subset V_{0}(\Gamma)$ obtained by adding the polynomial condition $f\left(\tau_{\gamma_{1}}\left(\chi_{\rho}\right)\right)=0 . A_{1}(\Gamma)$ is non-empty, since it contains $\rho_{1}$. Let $V_{1}(\Gamma)$ denote the irreducible component of $A_{1}(\Gamma)$ containing $\chi_{\rho_{1}}$. Note that any character in $V_{1}(\Gamma)$ will map $\gamma_{1}$ to an algebraic number (in fact to a root of $f$ ).

If $\operatorname{dim}\left(V_{1}(\Gamma)\right)=0$, then, as above, we have that $\rho_{1}$ is conjugate to an algebraic representation, and we are done. If $\operatorname{dim}\left(V_{1}(\Gamma)\right)>0$, then we can assume that $\tau_{\gamma_{2}}$ is non-constant on $V_{1}$, and then, as above, we can find an infinite irreducible representation $\rho_{2} \in V_{1}$ for which $\chi_{\rho_{2}}$ is algebraic. Then we form the set $V_{2}(\Gamma)$, and so on. Eventually, the process will terminate, when

i. $\operatorname{dim}\left(V_{m}(\Gamma)\right)=0$ for some $m$, in which case we will get an algebraic representation, or

ii. we have found an infinite irreducible representation $\rho$ such that $\chi_{\rho}\left(\gamma_{i}\right)$ is algebraic for all $i \leq n$. Since $\chi_{\rho}$ is a polynomial in the $\chi_{\rho}\left(\gamma_{i}\right)$ 's, then $\chi_{\rho}(\gamma)$ is algebraic for all $\gamma \in \Gamma$, and therefore by [Ba], $\rho$ is conjugate to an algebraic representation. 


\section{LEMMAS FROM GROUP THEORY AND NUMBER THEORY}

In this section we prove some lemmas which will be useful later, and we review the construction of the homomorphisms of $\Gamma$ into $P S L_{2}(\mathbb{C})$ alluded to in Section 3. For a more complete treatment of this construction, see [LR]. For background on algebraic number theory, see $[\mathrm{N}]$.

Let $\rho$ be an algebraic representation of $\Gamma$ (see Section 5), and let $\mathbb{Q}(\operatorname{tr} \rho(\Gamma))=\mathbb{Q}(\{\operatorname{tr} \rho(\gamma) \mid \gamma \in \Gamma\})$. Since $\rho$ is algebraic, this is a finite extension of $\mathbb{Q}$. It will be more convenient to work with the Galois closure, denoted $\overline{\mathbb{Q}(\operatorname{tr} \rho \Gamma)}$. This is also a finite extension, of degree $N$, say. Let $O$ denote the ring of integers of $\overline{\mathbb{Q}(\operatorname{tr} \rho(\Gamma))}$. It follows from the general theory of linear groups that for all but finitely many primes $p \in \mathbb{Z}$, there is a homomorphism $\phi_{p}: \Gamma \rightarrow P S L_{2}\left(\mathbb{F}_{p^{i}}\right)$, where $\mathbb{F}_{p^{i}}$ is the residue field of a prime ideal $P \subset O$ lying over $p$. In particular, if $p$ splits completely in $\overline{\mathbb{Q}(\operatorname{tr} \rho(\Gamma))}$ - i.e. factors into $N$ distinct prime ideals in $O$ - then $\phi_{p}$ maps into $P S L_{2}\left(\mathbb{F}_{p}\right)$.

Since we shall require maps into groups of the form $P S L_{2}\left(\mathbb{F}_{p}\right)$, it will be useful to know how many primes in $\mathbb{Z}$ split completely in $\overline{\mathbb{Q}(\operatorname{tr} \rho(\Gamma))}$. To give a precise answer requires the notion of natural density. if

Let $A$ be a set of primes in $\mathbb{Z}$. $A$ is said to have natural density $\delta$

$$
\lim _{t \rightarrow \infty}\left(\frac{\# \text { of primes in } A<t}{\# \text { of primes in } \mathbb{Z}<t}\right)=\delta .
$$

We may now state a simple version of the Tchebotarev Density Theorem (see [L] p. 128).

Theorem 6.1. (Tchebotarev density) Let $K$ be a Galois extension of $\mathbb{Q}$ of degree $N$, and let $P=\{$ primes in $\mathbb{Z}$ which split completely in $K\}$. Then the natural density of $P$ is $1 / N$. In particular, $P$ is infinite.

In fact, if $p$ splits completely, then $\phi_{p}$ will almost always surject onto $P S L_{2}\left(\mathbb{F}_{p}\right)$. The following theorem follows directly from the proof of Theorem 1.2 in [LR]:

Theorem 6.2. (Long-Reid) Let $\Gamma$ be a finitely generated group which admits an algebraic representation $\rho$. Let $P=\{$ primes in $\mathbb{Z}$ which split completely in $\overline{\mathbb{Q}(\operatorname{tr} \rho(\Gamma))}\}$, so that, for all but finitely many $p \in P$, the map $\phi_{p}: \Gamma \rightarrow P S L_{2}\left(\mathbb{F}_{p}\right)$ exists (see above). Then for all but finitely many $p \in P, \phi_{p}$ is a surjection. 
We will in fact require $\Gamma$ to surject onto a product of finite linear groups, prompting the following group theoretic lemma:

Lemma 6.3. Let $\Gamma$ be a group, and supppose $\Gamma$ surjects onto a sequence $G_{1}, \ldots, G_{n}$ of distinct, finite simple groups. Then $\Gamma$ surjects onto the direct product $\Pi_{i=1}^{n} G_{i}$.

Proof. We are given surjections $\phi_{i}: \Gamma \rightarrow G_{i}$.

Let $\phi_{1} \times \ldots \times \phi_{n}: \Gamma \rightarrow \Pi_{i=1}^{n} G_{i}$ denote the natural map induced by the $\phi_{i}$ 's. Let $H$ denote the image of $\Gamma$ under $\phi_{1} \times \ldots \times \phi_{n}$.

Let

$$
\text { (*) } \quad 1=N_{k} \triangleleft N_{k-1} \triangleleft \ldots \triangleleft N_{1} \triangleleft N_{0}=H
$$

be a chief series for $H$ - i.e. each $N_{i}$ is normal in $N_{i-1}$, and each quotient $N_{i-1} / N_{i}$ is simple. The Jordan-Holder Theorem (see [I], p. 132) guarantees that such a series exists, and that the quotient groups are unique up to re-ordering.

Let $\pi_{i}: H \rightarrow G_{i}$ be the natural projection map. Since $\phi_{i}$ surjects $\Gamma$ onto $G_{i}, \pi_{i}$ surjects $H$ onto $G_{i}$. Since $H / k e r \pi_{i} \cong G_{i}$ is simple, it follows that $H$ has another chief series in which $k e r \pi_{i}$ is the first term (again see $[\mathrm{I}]$ ). Therefore for each $i, G_{i}$ must appear as one of the quotients in the series $\left(^{*}\right)$. Therefore

$$
\begin{aligned}
|H| & \geq \Pi_{i=0}^{k-1}\left|N_{i} / N_{i+1}\right| \leq \Pi_{i=1}^{n}\left|G_{i}\right| \\
& =\left|\Pi_{i=1}^{n} G_{i}\right|
\end{aligned}
$$

Therefore $H=\prod_{i=1}^{n} G_{i}$, and so $\phi_{1} \times \ldots \times \phi_{n}$ is onto, proving the lemma.

Lemma 6.4. Let $K$ be a Galois extension of degree $N$ over $\mathbb{Q}$, and let $P=\{$ primes in $\mathbb{Z}$ which split completely in $K\}$. Let

$p_{1}, \ldots, p_{n-1} \in P$ with the property that $p_{1}>8 N$ and $p_{i}>2 p_{i-1}$ for all $1<i<n$. Then, given any two integers $r$ and $s$, there exist infinitely many primes in $P$ which are not congruent to $r$ or $s$ mod $p_{i}$ for any $i<n$.

Proof. By Theorem 6.1, $P$ has natural density $1 / N$ in $\mathbb{Z}$.

It follows from a more general version of Theorem 6.1 (see [L], p.128) that if $p_{i}$ does not divide $r$ or $s$, then the set of primes in $\mathbb{Z}$ which are congruent to $r \bmod p_{i}$ has natural density $1 /\left(p_{i}-1\right)$, as does the set congruent to $s \bmod p_{i}$.

Therefore, for large integers $m$ we have: 
$\frac{\mid \text { primes } p<m: p \in P \text { and } p \not \equiv r \text { or } s\left(\bmod p_{1}, \ldots \text { or } p_{n-1}\right) \mid}{\mid \text { primes }<m \mid}$

$=\frac{\mid \text { primes } p<m: p \in P \mid}{\mid \text { primes } p<m \mid}$

$$
-\frac{\mid \text { primes } p<m: p \equiv r \text { or } s\left(\bmod p_{1}, \ldots, \text { or } p_{n}\right) \mid}{\mid \text { primes } p<m \mid}
$$

$\geq \frac{1}{\mid \text { primes } p<m \mid}[\mid$ primes $p<m: p \in P \mid$

$-\mid$ primes $p<m: p \equiv r\left(\bmod p_{1}\right) \mid-\ldots$

$-\mid$ primes $p<m: p \equiv r\left(\bmod p_{n-1}\right) \mid$

$-\mid$ primes $p<m: p \equiv s\left(\bmod p_{1}\right) \mid$

$-\ldots-\mid$ primes $\left.p<m: p \equiv s\left(\bmod p_{n-1}\right) \mid\right]$

$=\frac{1}{N}-\frac{1}{p_{1}-1}-\ldots-\frac{1}{p_{n-1}-1}-\frac{1}{p_{1}-1}-\ldots-\frac{1}{p_{n-1}-1} \pm \epsilon$,

for some small $\epsilon$, by the above density statements.

$=\frac{1}{N}-\frac{2}{p_{1}-1}-\ldots-\frac{2}{p_{n-1}-1} \pm \epsilon$

$\geq \frac{1}{N}-\frac{2}{8 N}-\ldots-\frac{2}{2^{n+3} N} \pm \epsilon, \quad$ because $p_{1}>8 N, p_{i}>2 p_{i-1}$

$>\frac{1}{N}-\frac{1}{2 N} \pm \epsilon$

$=\frac{1}{2 N} \pm \epsilon$.

So the ratio is bounded away from zero, and therefore there must be infinitely many primes in $P$ which are not congruent to either $r$ or $s \bmod p_{i}$ for any $i<n$.

\section{Proving NONCONJUGaCY}

In Section 4 we showed how to construct elements $w_{n, i} \in \Gamma$ in the same trace class. Now we shall prove that these elements are pairwise non-conjugate.

It will be convenient to have the words written out in explicit form here:

$w_{1,1}=\left(a^{p_{1}-1+q_{1}} b^{-q_{1}}\right)^{k_{1}} a\left(a^{p_{1}-1+q_{1}} b^{-q_{1}}\right) a^{-1}$

$w_{1,2}=a\left(a^{p_{1}-1+q_{1}} b^{-q_{1}}\right)^{k_{1}} a^{-1}\left(a^{p_{1}-1+q_{1}} b^{-q_{1}}\right)$ 


$$
\begin{aligned}
w_{2,1}= & \left(w_{1,1}^{p_{2}-1+q_{2}} w_{1,2}^{-q_{2}}\right)^{k_{2}} w_{1,1}\left(w_{1,1}^{p_{2}-1+q_{2}} w_{1,2}^{-q_{2}}\right) w_{1,1}^{-1} \\
w_{2,2}= & w_{1,1}\left(w_{1,1}^{p_{2}-1+q_{2}} w_{1,2}^{-q_{2}}\right)^{k_{2}} w_{1,1}^{-1}\left(w_{1,1}^{p_{2}-1+q_{2}} w_{1,2}^{-q_{2}}\right) \\
w_{2,3}= & w_{2,1}^{[3]}=\left(w_{1,2}^{p_{2}-1+q_{2}} w_{1,1}^{-q_{2}}\right)^{k_{2}} w_{1,2}\left(w_{1,2}^{p_{2}-1+q_{2}} w_{1,1}^{-q_{2}}\right) w_{1,2}^{-1} \\
w_{n, 1}= & \left(w_{n-1,1}^{p_{n}-1+q_{n}} w_{n-1,2}^{-q_{n}}\right)^{k_{n}} w_{n-1,1}\left(w_{n-1,1}^{p_{n}-1+q_{n}} w_{n-1,2}^{-q_{n}}\right) w_{n-1,1}^{-1} \\
w_{n, 2}= & w_{n-1,1}\left(w_{n-1,1}^{p_{n}-1+q_{n}} w_{n-1,2}^{-q_{n}}\right)^{k_{n}} w_{n-1,1}^{-1}\left(w_{n-1,1}^{p_{n}-1+q_{n}} w_{n-1,2}^{-q_{n}}\right) \\
w_{n, 3}= & w_{n, 1}^{[3]}=W_{n}\left(w_{n-2,2}, w_{n-1,1}\right) \\
= & \left(w_{n-1,2}^{p_{n}-1+q_{n}} w_{n-1,1}^{-q_{n}}\right)^{k_{n}} w_{n-1,2}\left(w_{n-1,2}^{p_{n}-1+q_{n}} w_{n-1,1}^{-q_{n}}\right) w_{n-1,2}^{-1}, \text { for } n \geq 2 \\
w_{n, i}= & w_{n, 1}^{[i]} \\
= & {\left[\left(w_{n-1,1}^{[i-1]}\right)^{p_{n}-1+q_{n}}\left(w_{n-1,2}^{[i-1]}\right)^{-q_{n}}\right]^{k_{n}} } \\
& w_{n-1,1}^{[i-1]}\left[\left(w_{n-1,1}^{[i-1]}\right)^{p_{n}-1+q_{n}}\left(\left(w_{n-1,2}^{[i-1]}\right)^{-q_{n}}\right)\right]\left(w_{n-1,1}^{[i-1]}\right)^{-1}, \\
& \quad \text { for } n \geq 3 \text { and } 4 \leq i \leq n+1 .
\end{aligned}
$$

Let $\Gamma$ be as in the statement of Theorem 1.1, and let $\rho: \Gamma \rightarrow$ $S L_{2}(\mathbb{C})$ be the algebraic representation guaranteed by Lemma 5.1. Let $\mathbb{Q}(\operatorname{tr} \rho(\Gamma))$ be as in Section 6 , let $\overline{\mathbb{Q}(\operatorname{tr} \rho(\Gamma))}$ denote the Galois closure of $\mathbb{Q}(\operatorname{tr} \rho(\Gamma))$, and let $N=|\overline{\mathbb{Q}(\operatorname{tr} \rho(\Gamma))}: \mathbb{Q}|$. For primes $p$ which split completely in $\overline{\mathbb{Q}(\operatorname{tr} \rho(\Gamma))}$, let the map $\phi_{p}: \Gamma \rightarrow P S L_{2}\left(\mathbb{F}_{p}\right)$ be as discussed in Section 6 .

Let $Q=\left\{\right.$ primes $p \in \mathbb{Z}$ for which $\phi_{p}$ is a surjection $\}$. Note that $Q$ is infinite by Theorem 6.2. The proof of Theorem 1.1 follows from:

Proposition 7.1. The words $w_{n, i}$ can be chosen such that for every $n$ there exist $a_{n}, b_{n} \in \Gamma$ for which $w_{n, i}\left(a_{n}, b_{n}\right)$ is not conjugate to $w_{n, j}\left(a_{n}, b_{n}\right)$ or $w_{n, j}\left(a_{n}, b_{n}\right)^{-1}$ whenever $i \neq j$. Indeed, the words $w_{n, i}$ and elements $a_{n}, b_{n}$ can be chosen such that the following properties hold, for all $n$ :

$P 1(n):$ The primes $\left\{p_{1}, p_{2}, \ldots p_{n}\right\}$ are in $Q, p_{1}>8 N$, and $p_{i}>2 p_{i-1}$ for all $i \leq n$.

P2(n): For all $i \leq n, \phi_{p_{i}}\left(a_{n}\right)=\left[\begin{array}{cc}2 & 1 \\ 0 & 1 / 2\end{array}\right]$ and $\phi_{p_{i}}\left(b_{n}\right)=\left[\begin{array}{cc}2 & 0 \\ 0 & 1 / 2\end{array}\right]$. 
P3(n): For all $i \leq n$ and $j \leq n+1-i, \phi_{p_{i}}\left(w_{n, j}\left(a_{n}, b_{n}\right)\right)=i d$ and $\phi_{p_{i}}\left(w_{n, n+2-i}\left(a_{n}, b_{n}\right)\right)=\left[\begin{array}{ll}1 x \\ 0\end{array}\right] \neq i d$.

We say that $p_{i}$ "distinguishes" $w_{n, j}\left(a_{n}, b_{n}\right)$ from $w_{n, n+2-i}\left(a_{n}, b_{n}\right)$.

For example, taking $n=3$, we have that $p_{1}$ distinguishes $w_{3,4}$ from $w_{3,3}, w_{3,2}$ and $w_{3,1} ; p_{2}$ distinguishes $w_{3,3}$ from $w_{3,2}$ and $w_{3,1}$; and $p_{3}$ distinguishes $w_{3,2}$ from $w_{3,1}$.

Proof. (of Prop. 7.1) The proof is by induction. We begin by distinguishing $w_{1,1}$ from $w_{1,2}$. We must first pick the prime $p_{1}$ and the integers $q_{1}$ and $k_{1}$ which define $w_{1,1}$ and $w_{1,2}$.

Let $q_{1}=1$, let $p_{1}>\max \{8 N, 30\}$ be a prime in $Q$, and let $k_{1}=$ $p_{1}-4 . p_{1}, q_{1}$ and $k_{1}$ are now fixed, and will not change for the remainder of the proof. Note that P1(1) is immediately satisfied.

Let $a_{1} \in \phi_{p_{1}}^{-1}\left(\left[\begin{array}{ll}2 & 1 \\ 0 & 1 / 2\end{array}\right]\right)$ and $b_{1} \in \phi_{p_{1}}^{-1}\left(\left[\begin{array}{ll}2 & 0 \\ 0 & 1 / 2\end{array}\right]\right)$, so P2(1) is satisfied.

Note that the images of $a_{1}$ and $b_{1}$ under $\phi_{p_{1}}$ generate a semi-direct product of cyclic groups, that words of the form $\left[\begin{array}{ll}1 & x \\ 0 & 1\end{array}\right](x \neq 0)$ have order $p_{1}$, and that words of the form $\left[\begin{array}{cc}z & x \\ 0 & z^{-1}\end{array}\right](x \neq 0, z \neq 0,1)$ have order dividing $p_{1}-1$. This all follows from the structure theory of the groups $P S L_{2}\left(\mathbb{F}_{p}\right)$, and can be checked by explicit computation.

We have:

$$
\begin{aligned}
\phi_{p_{1}}\left(w_{1,1}(a, b)\right)= & {\left[\left(\begin{array}{cc}
2 & 1 \\
0 & 1 / 2
\end{array}\right)^{p_{1}}\left(\begin{array}{cc}
1 / 2 & 0 \\
0 & 2
\end{array}\right)\right]^{p_{1}-4} } \\
& \left(\begin{array}{cc}
2 & 1 \\
0 & 1 / 2
\end{array}\right)\left[\left(\begin{array}{cc}
2 & 1 \\
0 & 1 / 2
\end{array}\right)^{p_{1}}\left(\begin{array}{cc}
1 / 2 & 0 \\
0 & 2
\end{array}\right)\right]\left(\begin{array}{cc}
1 / 2 & -1 \\
0 & 2
\end{array}\right) \\
= & {\left[\left(\begin{array}{cc}
2 & 1 \\
0 & 1 / 2
\end{array}\right)\left(\begin{array}{cc}
1 / 2 & 0 \\
0 & 2
\end{array}\right)\right]^{p_{1}-4} } \\
& \left(\begin{array}{cc}
2 & 1 \\
0 & 1 / 2
\end{array}\right)\left[\left(\begin{array}{cc}
2 & 1 \\
0 & 1 / 2
\end{array}\right)\left(\begin{array}{cc}
1 / 2 & 0 \\
0 & 2
\end{array}\right)\right]\left(\begin{array}{cc}
1 / 2 & -1 \\
0 & 2
\end{array}\right) \\
& \operatorname{since}\left(\begin{array}{cc}
2 & 1 \\
0 & 1 / 2
\end{array}\right) \text { has order dividing } p_{1}-1 . \\
= & \left(\begin{array}{cc}
1 & 2 \\
0 & 1
\end{array}\right)\left(\begin{array}{cc}
2 & 1 \\
0 & 1 / 2
\end{array}\right)\left(\begin{array}{cc}
1 & 2 \\
0 & 1
\end{array}\right)\left(\begin{array}{cc}
1 / 2 & -1 \\
0 & 2
\end{array}\right) \\
= & \left(\begin{array}{ll}
1 & 2 p_{1}-8 \\
0 & 1
\end{array}\right)\left(\begin{array}{ll}
1 & 8 \\
0 & 1
\end{array}\right) \\
= & \left(\begin{array}{ll}
1 & 2 p_{1} \\
0 & 1
\end{array}\right) \\
= & \left(\begin{array}{ll}
1 & 0 \\
0 & 1
\end{array}\right) .
\end{aligned}
$$


However,

$$
\begin{aligned}
\phi_{p_{1}}\left(w_{1,2}(a, b)\right) & =\left(\begin{array}{cc}
2 & 1 \\
0 & 1 / 2
\end{array}\right)\left[\left(\begin{array}{cc}
2 & 1 \\
0 & 1 / 2
\end{array}\right)^{p_{1}}\left(\begin{array}{cc}
1 / 2 & 0 \\
0 & 2
\end{array}\right)\right]^{p_{1}-4}\left(\begin{array}{cc}
1 / 2 & -1 \\
0 & 2
\end{array}\right) \\
& =\left(\begin{array}{cc}
2 & 1 \\
0 & 1 / 2
\end{array}\right)\left[\begin{array}{cc}
1 / 2 & 0 \\
0 & 1 / 2
\end{array}\right)\left[\left(\begin{array}{cc}
2 & 1 \\
0 & 1 / 2
\end{array}\right)\left(\begin{array}{cc}
1 / 2 & 0 \\
0 & 2
\end{array}\right)\right]^{p_{1}-4}\left(\begin{array}{cc}
1 / 2 & -1 \\
0 & 2
\end{array}\right) \\
& \left.=\left(\begin{array}{cc}
2 & 1 \\
0 & 1 / 2
\end{array}\right)\left(\begin{array}{cc}
1 / 2 & 0 \\
0 & 2
\end{array}\right)\right] \\
& =\left(\begin{array}{cc}
1 & 2\left(p_{1}-4\right) \\
0 & 8\left(p_{1}-4\right) \\
0 & 1
\end{array}\right)\left(\begin{array}{cc}
1 & 2 \\
0 & 1 / 2 \\
0 & 1
\end{array}\right)\left(\begin{array}{cc}
1 & 2 \\
0 & 1
\end{array}\right) \\
& =\left(\begin{array}{cc}
1 & -30 \\
0 & 1
\end{array}\right) \\
& \neq\left(\begin{array}{cc}
1 & 0 \\
0 & 1
\end{array}\right) \text { since } p_{1}>30 .
\end{aligned}
$$

Therefore $p_{1}$ distinguishes $w_{1,1}\left(a_{1}, b_{1}\right)$ from $w_{1,2}\left(a_{1}, b_{1}\right)$, and P3(1) is satisfied.

Now, suppose that we have picked $a_{n-1}, b_{n-1} \in \Gamma$ and, for $i \leq n-1$, integers $k_{i}, p_{i}, q_{i}$ with corresponding words $w_{i, j}$, so that the following is true:

$\mathrm{P} 1(\mathrm{n}-1)$ : The primes $\left\{p_{1}, \ldots, p_{n-1}\right\}$ are in $Q$, and $p_{i}>2 p_{i-1}$ for all $i \leq n-1$.

$\mathrm{P} 2(\mathrm{n}-1)$ : For all $i \leq n-1, \phi_{p_{i}}\left(a_{n-1}\right)=\left[\begin{array}{cc}2 & 1 \\ 0 & 1 / 2\end{array}\right]$ and $\phi_{p_{i}}\left(b_{n-1}\right)=\left[\begin{array}{cc}2 & 0 \\ 0 & 1 / 2\end{array}\right]$.

P3(n-1): For all $i \leq n-1$ and $j \leq n-i, \phi_{p_{i}}\left(w_{n-1, j}\left(a_{n-1}, b_{n-1}\right)\right)=i d$ and $\phi_{p_{i}}\left(w_{n-1, n+1-i}\left(a_{n-1}, b_{n-1}\right)\right)=\left[\begin{array}{ll}1 & x \\ 0 & 1\end{array}\right] \neq i d$.

We shall show how to pick $k_{n}, q_{n}, p_{n}, a_{n}$ and $b_{n}$ so that Properties $\mathrm{P} 1(\mathrm{n}), \mathrm{P} 2(\mathrm{n})$ and P3(n) are satisfied.

Since $Q$ is infinite by Theorem 6.2, Property $\mathrm{P} 1(\mathrm{n})$ may be satisfed simply by taking $p_{n}$ to be large enough. 
Observe that, by Lemma 6.3, $\Gamma$ surjects onto $\prod_{i=1}^{n} P S L_{2}\left(\mathbb{F}_{p_{i}}\right)$. Therefore we can satisfy Property $\mathrm{P} 2(\mathrm{n})$ by picking $a_{n} \in \bigcap_{i=1}^{n} \phi_{p_{i}}^{-1}\left(\left[\begin{array}{cc}2 & 1 \\ 0 & 1 / 2\end{array}\right]\right)$ and $b_{n} \in \bigcap_{i=1}^{n} \phi_{p_{i}}^{-1}\left(\left[\begin{array}{cc}2 & 0 \\ 0 & 1 / 2\end{array}\right]\right)$.

To satisfy Property P3(n), we must show that, for all $i \leq n$ and $j \leq n+1-i, \phi_{p_{i}}\left(w_{n, j}\right)=i d$ and $\phi_{p_{i}}\left(w_{n, n+2-i}\right)=\left[\begin{array}{ll}1 & x \\ 0 & 1\end{array}\right] \neq i d$.

Let $q_{n}=\Pi_{j=1}^{n-1} p_{j}\left(p_{j}-1\right)$.

We will break the proof up into two cases; first we will see what happens when we reduce by the primes $p_{i}, i<n$, which we have already picked, and then we show how to pick $p_{n}$.

Case 1: $i<n$.

$\phi_{p_{i}}\left(a_{n}\right)=\left[\begin{array}{cc}2 & 1 \\ 0 & 1 / 2\end{array}\right]$ and $\phi_{p_{i}}\left(b_{n}\right)=\left[\begin{array}{cc}2 & 0 \\ 0 & 1 / 2\end{array}\right]$ are contained in the subgroup $B \subset P S L_{2}\left(\mathbb{F}_{p_{i}}\right)$ consisting of matrices whose lower left entry is 0 . The order of $B$ is $p_{i}\left(p_{i}-1\right) / 2$, which divides $q_{n}$. Therefore, $\phi_{p_{i}}\left(U\left(a_{n}, b_{n}\right)^{q_{n}}\right)=i d$, where $U$ is any word on two letters. So we have, for $j \leq n+1-i$ :

Case 1a: $j=1$

$$
\begin{aligned}
\phi_{p_{i}}\left(w_{n, 1}\right) & =\phi_{p_{i}}\left(\left[w_{n-1,1}^{p_{n}-1+q_{n}} w_{n-1,2}^{-q_{n}}\right]^{k_{n}} w_{n-1,1}\left[w_{n-1,1}^{p_{n}-1+q_{n}} w_{n-1,2}^{-q_{n}}\right] w_{n-1,1}^{-1}\right. \\
& =\phi_{p_{i}}\left(w_{n-1,1}^{\left(p_{n}-1\right)\left(k_{n}+1\right)}\right) \\
& =i d, \quad \text { by Property P3(n-1). }
\end{aligned}
$$

Case 1b: $j=2$

$$
\begin{aligned}
\phi_{p_{i}}\left(w_{n, 2}\right) & =\phi_{p_{i}}\left(w_{n-1,1}\left[w_{n-1,1}^{p_{n}-1+q_{n}} w_{n-1,2}^{-q_{n}}\right]^{k_{n}} w_{n-1,1}^{-1}\left[w_{n-1,1}^{p_{n}-1+q_{n}} w_{n-1,2}^{-q_{n}}\right]\right) \\
& =\phi_{p_{i}}\left(w_{n-1,1}^{\left(p_{n}-1\right)\left(k_{n}+1\right)}\right) \\
& =i d, \quad \text { by Property P3(n-1). }
\end{aligned}
$$

Case 1c: $j=3$

$$
\begin{aligned}
\phi_{p_{i}}\left(w_{n, 3}\right)= & \phi_{p_{i}}\left(w_{n, 1}^{[3]}\right) \\
& \left(\text { recall the definition of } w_{n, i}^{[j]} \text { from Section } 4\right) \\
= & \phi_{p_{i}}\left(W_{n}\left(w_{n-2,2}, w_{n-1,1}\right)\right) \\
= & \phi_{p_{i}}\left(\left[w_{n-1,2}^{p_{n}-1+q_{n}} w_{n-1,1}^{-q_{n}}\right]^{k_{n}} w_{n-1,2}\left[w_{n-1,2}^{p_{n}-1+q_{n}} w_{n-1,1}^{-q_{n}}\right] w_{n-1,2}^{-1}\right) \\
= & \phi_{p_{i}}\left(w_{n-1,2}^{\left(p_{n}-1\right)\left(k_{n}+1\right)}\right) \\
= & i d, \quad \text { by Property P3(n-1), since } j-1=2 \leq n-i
\end{aligned}
$$


Case 1d: $4 \leq j \leq n+1-i$

$$
\begin{aligned}
\phi_{p_{i}}\left(w_{n, j}\right) & =\phi_{p_{i}}\left(w_{n, 1}^{[j]}\right) \\
& =\phi_{p_{i}}\left(\left[\left(w_{n-1,1}^{[j-1]}\right)^{p_{n}-1+q_{n}}\left(w_{n-1,2}^{[j-1]}\right)^{-q_{n}}\right]^{k_{n}}\right. \\
& \left.\quad w_{n-1,1}^{[j-1]}\left[\left(w_{n-1,1}^{[j-1]}\right)^{p_{n}-1+q_{n}}\left(w_{n-1,2}^{[j-1]}\right)^{-q_{n}}\right]\left(w_{n-1,1}^{[j-1]}\right)^{-1}\right) \\
& =\phi_{p_{i}}\left(\left(w_{n-1,1}^{[j-1]}\right)^{\left(p_{n}-1\right) k_{n}+p_{n}-1}\right) \\
& =\phi_{p_{i}}\left(w_{n-1, j-1}^{\left(p_{n}-1\right)\left(k_{n}+1\right)}\right) \\
& =i d, \quad \text { by Property P3(n-1), since } j-1 \leq n-i .
\end{aligned}
$$

However,

$$
\begin{aligned}
& \phi_{p_{i}}\left(w_{n, n+2-i}\right)=\phi_{p_{i}}\left(w_{n, 1}^{[n+2-i]}\right) \text {, since } i \leq n-1 \text {, so } n+2-i \geq 3 \text {. } \\
& (\text { for } i<n-1)=\phi_{p_{i}}\left(\left[\left(w_{n-1,1}^{[n+1-i]}\right)^{p_{n}-1+q_{n}}\left(w_{n-1,2}^{[n+1-i]}\right)^{-q_{n}}\right]^{k_{n}} w_{n-1,1}^{[n+1]}\right. \\
& \left.\left[\left(w_{n-1,1}^{[n+1-i]}\right)^{p_{n}-1+q_{n}}\left(w_{n-1,2}^{[n+1-i]}\right)^{-q_{n}}\right]\left(w_{n-1,1}^{[n+1-i]}\right)^{-1}\right) \\
& =\phi_{p_{i}}\left(\left[w_{n-1,1}^{[n+1-i]}\right]^{\left(p_{n}-1\right)\left(k_{n}+1\right)}\right) \\
& =\phi_{p_{i}}\left(w_{n-1, n+1-i}^{\left(p_{n}-1\right)\left(k_{n}+1\right)}\right) \\
& =\left(\begin{array}{cc}
1 & \left(p_{n}-1\right)\left(k_{n}+1\right) x \\
0 & 1
\end{array}\right) \\
& \text { where } x \neq 0 \text { by Property P3(n-1). } \\
& (\text { for } i=n-1)=\phi_{p_{n-1}}\left(w_{n, 1}^{[3]}\right) \\
& =\phi_{p_{n-1}}\left(\left[w_{n-1,2}^{p_{n}-1+q_{n}} w_{n-1,1}^{-q_{n}}\right]^{k_{n}}\right. \\
& \left.w_{n-1,2}\left[w_{n-1,2}^{p_{n}-1+q_{n}} w_{n-1,1}^{-q_{n}}\right] w_{n-1,2}^{-1}\right) \\
& =\phi_{p_{n-1}}\left(w_{n-1,2}^{\left(p_{n}-1\right)\left(k_{n}+1\right)}\right) \\
& =\left(\begin{array}{cc}
1 & \left(p_{n}-1\right)\left(k_{n}+1\right) x \\
0 & 1
\end{array}\right) \text {, } \\
& \text { where } x \neq 0 \text { by Property P3(n-1). }
\end{aligned}
$$

So to satisfy Property P3(n), we must pick $p_{n} \in Q$ and $k_{n}$ such that:

(I) $p_{i}$ does not divide $k_{n}+1$ or $p_{n}-1$ for $i<n$.

Let $m$ be the sum of the exponents on $a_{n}$ and $b_{n}$ in $w_{n-1,1}\left(a_{n}, b_{n}\right)$. We shall also require:

(II) $p_{n}>\max \left\{2^{4 m}, q_{n}\right\}$ 
We set $k_{n}=p^{n}-2^{2 m}$.

We claim that we can pick $p_{n}$ to satisfy properties $(I)$ and $(I I)$. Indeed, $(I)$ is equivalent to the statement

$\left(I^{\prime}\right) p_{n} \not \equiv 1$ or $2^{2 m}-1\left(\bmod p_{i}\right)$ for any $i<n$.

Property $\mathrm{P} 1(\mathrm{n}-1)$ guarantees that $p_{i}>2 p_{i-1}$ for all $i<n$ and $p_{1}>8 N$, so Lemma 6.4 implies that $\left(I^{\prime}\right)$ can be satisfied by infinitely many primes $p \in Q$. Therefore we can pick an arbitrarily large prime $p_{n}$ to satisfy $(I)$ and $(I I)$, and still satisfy Property $\mathrm{P} 1(\mathrm{n})$.

This concludes the proof in Case 1.

Case 2: $i=n$

We now show how to distinguish $w_{n, 1}$ from $w_{n, 2}$.

Since $\phi_{p_{n}}\left(a_{n}\right)=\left[\begin{array}{cc}2 & 1 \\ 0 & 1 / 2\end{array}\right]$ and $\phi_{p_{n}}\left(b_{n}\right)=\left[\begin{array}{cc}2 & 0 \\ 0 & 1 / 2\end{array}\right]$, it is easy to see that $\phi_{p_{n}}\left(w_{n-1,1}\right)$ will have the form $\left[\begin{array}{cc}2^{m} & w \\ 0 & 2^{-m}\end{array}\right]$. Note $2^{m} \neq 1\left(\bmod p_{n}\right)$, since $p_{n}>2^{4 m}$. Therefore, $\phi_{p_{n}}\left(w_{n-1,1}^{p_{n}-1}\right)=i d$, and we have:

$$
\begin{gathered}
\phi_{p_{n}}\left(w_{n, 1}\right)=\phi_{p_{n}}\left(\left(w_{n-1,1}^{p_{n}-1+q_{n}} w_{n-1,2}^{-q_{n}}\right)^{k_{n}}\right. \\
\left.w_{n-1,1}\left(w_{n-1,1}^{p_{n}-1+q_{n}} w_{n-1,2}^{-q_{n}}\right) w_{n-1,1}^{-1}\right) \\
=\phi_{p_{n}}\left(\left(w_{n-1,1}^{q_{n}} w_{n-1,2}^{-q_{n}}\right)^{k_{n}} w_{n-1,1}\left(w_{n-1,1}^{q_{n}} w_{n-1,2}^{-q_{n}}\right) w_{n-1,1}^{-1}\right) .
\end{gathered}
$$

Lemma 7.2. If $p_{n}$ is chosen to be large enough, then

$$
\phi_{p_{n}}\left(w_{n-1,1}^{q_{n}} w_{n-1,2}^{-q_{n}}\right)=\left(\begin{array}{ll}
1 & z \\
0 & 1
\end{array}\right),
$$

where $z \neq 0$.

Proof. Recall that $p_{n-1}$ distinguishes $w_{n-1,1}$ from $w_{n-1,2}$. Therefore, $w_{n-1,1}(\bar{a}, \bar{b}) \neq w_{n-1,2}(\bar{a}, \bar{b})$ for $\bar{a}=\left[\begin{array}{cc}2 & 1 \\ 0 & 1 / 2\end{array}\right], \bar{b}=\left[\begin{array}{ll}1 & 1 \\ 0 & 1\end{array}\right] \in P S L_{2}\left(\mathbb{F}_{p_{n-1}}\right)$.

Now let $\mathbb{Z}(1 / 2)$ denote the ring obtained by adjoining $1 / 2$ to $\mathbb{Z}$, and let $\tilde{a}=\left[\begin{array}{cc}2 & 1 \\ 0 & 1 / 2\end{array}\right], \tilde{b}=\left[\begin{array}{ll}1 & 1 \\ 0 & 1\end{array}\right] \in P S L_{2}(\mathbb{Z}(1 / 2))$. There is a well-defined reduction map $\theta_{p_{n-1}}: P S L_{2}(\mathbb{Z}(1 / 2)) \rightarrow P S L_{2}\left(\mathbb{F}_{p_{n-1}}\right)$, since $p_{n-1} \neq 2$. Then

$$
\begin{aligned}
\theta_{p_{n-1}}\left(w_{n-1,1}(\tilde{a}, \tilde{b})\right) & =w_{n-1,1}(\bar{a}, \bar{b}) \\
& \neq w_{n-1,2}(\bar{a}, \bar{b})=\theta_{p_{n-1}}\left(w_{n-1,2}(\tilde{a}, \tilde{b})\right)
\end{aligned}
$$


so $w_{n-1,1}(\tilde{a}, \tilde{b}) \neq w_{n-1,2}(\tilde{a}, \tilde{b})$ in $P S L_{2}(\mathbb{Z}(1 / 2))$.

Using the definition of $w_{n-1, i}$ one may easily verify that the words have the following forms:

$$
\begin{array}{r}
w_{n-1,1}(\tilde{a}, \tilde{b})=\left(\begin{array}{cc}
2^{m} & x \\
0 & 2^{-m}
\end{array}\right) \\
\left.w_{n-1,2}(\tilde{a}, \tilde{b})\right)=\left(\begin{array}{cc}
2^{m} & y \\
0 & 2^{-m}
\end{array}\right), \text { where } x \neq y .
\end{array}
$$

Then we compute:

$$
\begin{aligned}
& \left(w_{n-1,1}(\tilde{a}, \tilde{b})\right)^{q_{n}}=\left(\begin{array}{cc}
2^{m} & x \\
0 & 2^{-m}
\end{array}\right)^{q_{n}} \\
& =\left(\begin{array}{cc}
2^{m q_{n}} x\left(2^{m\left(q_{n}-1\right)}+2^{m\left(q_{n}-3\right)}+\ldots+2^{m\left(3-q_{n}\right)}+2^{m\left(1-q_{n}\right)}\right) \\
0 & 2^{-m q_{n}}
\end{array}\right) \\
& \neq\left(\begin{array}{cc}
2^{m q_{n}} y\left(2^{m\left(q_{n}-1\right)}+2^{m\left(q_{n}-3\right)}+\ldots+2^{m\left(3-q_{n}\right)}+2^{m\left(1-q_{n}\right)}\right) \\
0 & 2^{-m q_{n}}
\end{array}\right) \\
& =\left(\begin{array}{cc}
2^{m} & y \\
0 & 2^{-m}
\end{array}\right)^{q_{n}} \\
& =\left(w_{n-1,2}(\tilde{a}, \tilde{b})\right)^{q_{n}} .
\end{aligned}
$$

Then for a large enough prime $p_{n}$, these words are different mod $p_{n}$ :

$$
\begin{aligned}
\phi_{p_{n}}\left(w_{n, 1}(a, b)\right)^{q_{n}} & =\theta_{p_{n}}\left(w_{n, 1}(\tilde{a}, \tilde{b})\right)^{q_{n}} \\
& =\left(\begin{array}{cc}
v & x^{\prime} \\
0 & 1 / v
\end{array}\right), \text { for some } v \\
\phi_{p_{n}}\left(w_{n, 2}(a, b)\right)^{q_{n}} & =\theta_{p_{n}}\left(w_{n, 2}(\tilde{a}, \tilde{b})\right)^{q_{n}} \\
& =\left(\begin{array}{cc}
v & y^{\prime} \\
0 & 1 / v
\end{array}\right), \text { where } x^{\prime} \neq y^{\prime} \in \mathbb{F}_{p_{n}} .
\end{aligned}
$$

Then

$$
\begin{aligned}
\phi_{p_{n}}\left(w_{n, 1}^{q_{n}} w_{n, 2}^{-q_{n}}\right) & =\left(\begin{array}{cc}
1 v\left(x^{\prime}-y^{\prime}\right) \\
0 & 1
\end{array}\right) \\
& \neq i d,
\end{aligned}
$$

completing the proof of Lemma 7.2 .

Then, returning to Equation 2, we have

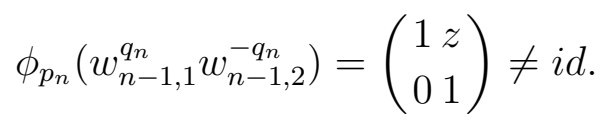


So,

$$
\begin{aligned}
\phi_{p_{n}}\left(w_{n, 1}\right) & =\left(\begin{array}{cc}
1 & k_{n} z \\
0 & 1
\end{array}\right)\left(\begin{array}{cc}
2^{m} & w \\
0 & 2^{-m}
\end{array}\right)\left(\begin{array}{ll}
1 & z \\
0 & 1
\end{array}\right)\left(\begin{array}{cc}
2^{-m} & -w \\
0 & 2^{m}
\end{array}\right) \text { for some } w . \\
& =\left(\begin{array}{cc}
1 & k_{n} z \\
0 & 1
\end{array}\right)\left(\begin{array}{cc}
1 & 2^{2 m} z \\
0 & 1
\end{array}\right) \\
& =\left(\begin{array}{cc}
1 & \left(k_{n}+2^{2 m}\right) z \\
0 & 1
\end{array}\right) \\
& =\left(\begin{array}{cc}
1 & \left(p_{n}-2^{2 m}+2^{2 m}\right) z \\
0 & 1
\end{array}\right), \text { by our choice of } k_{n} . \\
& =\left(\begin{array}{ll}
1 & 0 \\
0 & 1
\end{array}\right) .
\end{aligned}
$$

However:

$$
\begin{aligned}
\phi_{p_{n}}\left(w_{n, 2}\right) & =\phi_{p_{n}}\left(w_{n-1,1}\left(w_{n-1,1}^{p_{n}-1+q_{n}} w_{n-1,2}^{-q_{n}}\right)^{k_{n}} w_{n-1,1}^{-1}\left(w_{n-1,1}^{p_{n}-1+q_{n}} w_{n-1,2}^{-q_{n}}\right)\right) \\
& =\phi_{p_{n}}\left(w_{n-1,1}\left(w_{n-1,1}^{q_{n}} w_{n-1,2}^{-q_{n}}\right)^{k_{n}} w_{n-1,1}^{-1}\left(w_{n-1,1}^{q_{n}} w_{n-1,2}^{-q_{n}}\right)\right) \\
& =\left(\begin{array}{cc}
2^{m} & w \\
0 & 2^{-m}
\end{array}\right)\left(\begin{array}{cc}
1 & k_{n} z \\
0 & 1
\end{array}\right)\left(\begin{array}{cc}
2^{-m} & -w \\
0 & 2^{m}
\end{array}\right)\left(\begin{array}{cc}
1 & z \\
0 & 1
\end{array}\right) \\
& =\left(\begin{array}{cc}
1 & 2^{2 m} k_{n} z+z \\
0 & 1
\end{array}\right) \\
& =\left(\begin{array}{cc}
1 & \left(2^{2 m}\left(p_{n}-2^{2 m}\right)+1\right) z \\
0 & 1
\end{array}\right) . \\
& =\left(\begin{array}{cc}
1 & \left(-2^{4 m}+1\right) z \\
0 & 1
\end{array}\right) .
\end{aligned}
$$

Since $p_{n}>2^{4 m},-2^{4 m}+1 \neq 0\left(\bmod p_{n}\right)$. Also, $z \neq 0$, so $\phi_{p_{n}}\left(w_{n, 2}\right) \neq 0$. Hence Property P3(n) is satisfied, and we are done.

\section{REFERENCES}

[A] J. Anderson, "A brief survey of the deformation theory of Kleinian groups", preprint.

[Ba] H. Bass, Groups of integral representation type, Pacific J. Math. 86 (1980), 15-51.

[Br] R. Brooks, "Circle packings and co-compact extensions of Kleinian groups", Invent. Math. 86 (1986), 461-469. 
[CS] M. Culler and P. B. Shalen, "Varieties of group representations and splittings of 3-manifolds", Ann. Math. 117 (1983), 109-146.

[GR] D. Ginzburg and Z. Rudnick, "Stable multiplicities in the length spectrum of Riemann surfaces", Isr. J. Math. 104 (1998), 129-144.

[H] R. D. Horowitz, "Characters of free groups represented in the two-dimensional special linear group", Comm. Pure Appl. Math 25 (1972), 635-649.

[I] I. M. Isaacs, Algebra: a Graduate Course, Brooks/Cole, 1994.

[L] R. L. Long, Algebraic Number Theory, Marcel Dekker, 1977.

[LR] D. D. Long and A.W. Reid, "Simple quotients of Hyperbolic 3-manifold groups", Proc. Amer. Math. Soc. 126, (1998), 877-880.

[M] J. Marklof, "On multiplicities in length spectra of arithmetic hyperbolic three-orbifolds", Nonlinearity 9 (1996), 517-536.

[N] W. Narkiewicz, Algebraic Numbers, Polish Scientific Publishers, Warsaw, 1974.

[R] B. Randol, "The length spectrum of a Riemann surface is always of unbounded multiplicity", Proc. Amer. Math. Soc. 78 (1980), 455-456.

[Sar] P. Sarnak, "Arithmetic quantum chaos", The Schur Lectures (1992), Israel Mathematical Conference Proceedings Vol. 8, 1995.

[Sc] P. Scott, "Compact submanifolds of 3-manifolds", J. London Math. Soc. 7 (1973), 246-250.

[Su] M. Suzuki, Group Theory I, Springer-Verlag, 1982.

[T] W. Thurston, Geometry and Topology of Hyperbolic 3-manifolds, mimeographed lecture notes, 1978. 
Department of Mathematics

University of Texas at Austin

Austin TX 78712

masters@math.utexas.edu

Current Address:

Department of Mathematics

Rice University

Houston TX 77005-1892

masters@math.rice.edu 